\title{
Diffusion Tensor Analysis of Pediatric Multiple Sclerosis and Clinically Isolated Syndromes
}

\author{
M.S. Vishwas, B.C. Healy, R. Pienaar, M.P. Gorman, P.E. Grant, and T. Chitnis
}

\begin{abstract}
BACKGROUND AND PURPOSE: DTI has shown focal and diffuse white matter abnormalities in adults and children with MS. Here we explore whether DTI abnormalities are present at the time of a first attack or CIS in children and whether early DTI features can predict the development of MS.
\end{abstract}

\begin{abstract}
MATERIALS AND METHODS: We assessed region-of-interest and tract-based mean ADC and mean FA values for 3 major white matter pathways and NAWM in 20 children with MS, 27 children with forms of CIS, and controls. Tracts were selected by using standard region-of-interest placements on color FA maps. Identical ROIs were placed in the NAWM on $b=0$ T2-weighted images to ensure that both ROIs and resulting tracts passed through NAWM. Conventional MR imaging characteristics were assessed by visual inspection. Statistical analysis compared FA and ADC values between groups by a $t$ test. Logistic regression assessed the predictive value of DTI measures and published conventional MR imaging measures for conversion from CIS to MS.

RESULTS: In pediatric patients with MS, all white matter pathways and analysis confined to the NAWM demonstrated higher mean ADC values and lower mean FA than in controls. In contrast, there were no significant differences in mean ADC and mean FA of white matter pathways in all CIS cohorts compared with controls. In the CIS cohort, none of the DTI measures in white matter pathways or in NAWM were significantly associated with conversion to RRMS in univariate or multivariate models $(P>.05$ in all models).
\end{abstract}

CONCLUSIONS: There are significant anisotropic abnormalities in the NAWM of major tracts in children with MS. In contrast, there were no significant changes in pediatric patients with CIS compared with controls at baseline. DTI measures did not predict conversion to MS. The period between CIS and conversion to pediatric MS may represent a window of opportunity for the prevention of diffuse damage in the CNS and potentially progressive disability.

ABBREVIATIONS: CIS = clinically isolated syndrome; FA = fractional anisotropy; KIDMUS = Kids with MS; LAF = long-association fiber; MCIS = monofocal clinically isolated syndrome; NAWM = normal-appearing white matter; $\mathrm{PCIS}=$ polyfocal clinically isolated syndrome; $\mathrm{PCISWE}=$ polyfocal clinically isolated syndrome with encephalopathy; PCISWOE = polyfocal clinically isolated syndrome without encephalopathy; PLIC = posterior limb of the internal capsule; RRMS = relapsingremitting MS

$\mathrm{t}$ is estimated that $5 \%$ of all MS cases begin in childhood. ${ }^{1-3}$ Children with MS accumulate significant disability at an early age, ${ }^{2,4}$ experience more frequent relapses than those with adultonset MS, ${ }^{5}$ and demonstrate significant cognitive deficits. ${ }^{6,7}$ These findings, along with high T2 lesion load at the time of di-

Received December 4, 2011; accepted after revision April 18.

From Partners Pediatric Multiple Sclerosis Center (M.S.V., M.P.G., T.C.), Massachusetts General Hospital for Children, Boston, Massachusetts; Athinoula A. Martinos Center for Biomedical Imaging (M.S.V., R.P., P.E.G.), Massachusetts General Hospital, Harvard Medical School, Boston, Massachusetts; and Biostatistics Center (B.C.H.),

Massachusetts General Hospital, Boston, Massachusetts.

Dr Brian Healy conducted all bio-statistical analysis.

This work was supported by the Pediatric Multiple Sclerosis Centers of Excellence Grant from the National Multiple Sclerosis Society to T.C.

Please address correspondence to Tanuja Chitnis, MD, Partners Pediatric Multiple Sclerosis Center, Massachusetts General Hospital, ACC-708, 55 Fruit St, Boston, MA 02114; e-mail: tchitnis@partners.org agnosis, ${ }^{8}$ suggest that significant pathologic damage is present in pediatric MS.

Using DTI and diffusion tractography, we have recently demonstrated increased mean ADC and decreased mean FA in 3 major white matter pathways (interhemispheric, projection, and intrahemispheric) in children and adolescents with established MS. These findings occurred both in tracts with visibly involved focal lesions and in NAWM defined as tracts without discernible focal lesions, ${ }^{9}$ indicating that pathologic processes in pediatric MS diffusely involve the white matter.

Up to $80 \%$ of children presenting with clinically isolated syndromes, including acute disseminated encephalomyelitis, may

Indicates article with supplemental on-line table.

Indicates article with supplemental on-line figures.

http://dx.doi.org/10.3174/ajnr.A3216

AJNR Am J Neuroradiol 34:417-23 Feb 2013 www.ajnr.org 
eventually develop MS. ${ }^{10}$ Many of these children have visible MR imaging lesions ${ }^{11}$; however, it is unknown whether diffuse damage to the NAWM is present at the time of a first clinical episode. In addition, MR imaging features, such as the presence of welldefined periventricular and corpus callosum lesions and Barkhof criteria, are specific but insensitive predictors of the development of MS after a first attack in childhood. ${ }^{11,12}$ Advanced imaging techniques have not been evaluated in assessing future risk of MS in children with an initial demyelinating event.

In the present study, we compared DTI abnormalities in the major white matter pathways (interhemispheric, projection, and intrahemispheric) in pediatric patients with MS and subtypes of clinically isolated syndromes, including acute disseminated encephalomyelitis, to determine the earliest indication and extent of abnormalities in major white matter pathways and whether these changes may be predictive of the development of MS or chronic demyelinating disease. We also assessed the predictive value of conventional MR imaging criteria for the development of MS.

\section{MATERIALS AND METHODS \\ Patients}

We conducted a retrospective search of the central data base at Massachusetts General Hospital for Children for patients younger than 18 years of age with the diagnoses of CIS, acute disseminated encephalomyelitis, and RRMS based on the diagnostic criteria proposed by the International Pediatric MS Study Group. ${ }^{13} \mathrm{~Pa}-$ tients must have had $\geq 1$ MRI performed at Massachusetts General Hospital with DTI sequences. The Internal Review Board for Human Studies of Partners approved the use of data from human subjects.

\section{Pediatric MS}

Twenty patients younger than 18 years of age with established RRMS and an MR imaging examination including a DTI sequence performed during a 30-day relapse-free period were included in the study.

\section{Clinically Isolated Syndrome}

Patients younger than 18 years of age with a first clinically isolated syndrome and DTI performed within 6 months were included in the study. Twenty-seven patients were included on the basis of the inclusion criteria. Patients were subcategorized on the basis of the following proposed definitions: ${ }^{12}$

\section{MCIS}

MCIS is a monofocal attack clinically ascribed to demyelination in 1 area of the CNS ( $n=9 / 27)$ : the optic nerve, brain stem, cerebral hemispheres, cerebellum, or spinal cord.

\section{PCIS}

PCIS is a polyregional attack, clinically ascribed to multiple CNS areas. We avoided the use of the term acute disseminated encephalomyelitis because of incomplete consensus on this diagnosis. ${ }^{12}$ Hence, we classified the patients with PCIS into 2 subgroups: PCISWOE and PCISWE. Encephalopathy was defined as an altered level of consciousness or change in higher mental status within 3 days of the first demyelinating event unrelated to seizures or antiepileptic treatment. Nine patients were included in the
PCISWOE group, and 9 patients were included in the PCISWE group. There were 27 unique patients with CIS with baseline MR imaging. Six of these patients converted to RRMS and were included in the 20 patients with RRMS described above.

\section{Controls}

Age-, sex-, and DTI technique-matched healthy controls for each case were obtained from the Massachusetts General Hospital clinical data base. Children with headache as a presenting symptom, normal clinical examination findings, and grossly normal brain MR imaging findings on conventional T1 and T2 sequences and normal findings on diffusion sequences were included for comparison. There was no overlap of control images for the baseline MR imaging analysis among all 4 groups.

\section{MR Imaging Acquisition}

All scans were performed on 1.5T Signa HDx or Excite scanners (GE Healthcare, Milwaukee, Wisconsin). All patients had conventional proton attenuation-weighted and T2-weighted images $(\mathrm{TR} / \mathrm{TE}=3350-7260 / 97-109 \mathrm{~ms}$ and $4-$ to 5 -mm-thick sections), and DTI parameters used were as follows: 19 patients with $1 b=0 ; 10$ patients with $3 b=0 ; 18$ patients with $5 b=0$ (TR/ $\mathrm{TE}=3000-6570 / 83-97 \mathrm{~ms} ; 23$ contiguous $6-\mathrm{mm}$-thick sections to 52 contiguous 2.2 -mm-thick sections; voxel size, $1.72 \times 1.72 \times$ 6 to $0.86 \times 0.86 \times 2.2$; and $6-29$ gradient directions at $b=1000$ $\mathrm{s} / \mathrm{mm}^{2}$ ). All control MRIs were matched for scanner subtype, section thickness, $b=0$, and gradient directions for both baseline MR imaging and longitudinal MR imaging analysis.

\section{Image Processing, Tract Reconstruction, and Image Analysis}

A custom-designed shell script collected DICOM images, extracted gradient tables, determined the number of B0 volumes, and converted data to Neuroimaging Informatics Technology Initiative format (http://nifti.nimh.nih.gov/nifti-1/). ${ }^{14}$ The processing script interacted with the diffusion toolkit (http://trackvis.org/ blog/tag/diffusion-toolkit/) and reconstruction without user input, and raw data were reconstructed, outputting a set of tensor images, DWI, ADC, and FA maps. Reconstructed data were converted into tracts by using the standard fiber assignment by continuous tracking ${ }^{14}$ algorithm with an angle threshold set at $35^{\circ}$. As in our previous study, ${ }^{9}$ we used a DWI mask to remove CSF but did not use an FA threshold for tract reconstruction because the standard FA threshold of 0.2 would drop out tracts passing through some lesions and bias our results. Region-of-interest placement ${ }^{15-17}$ and fiber tract data analysis ${ }^{15-17}$ were performed on Track-Vis (www.trackvis.org). ${ }^{9}$ The seed points were placed, manually defining a region of interest by referring to standard color FA maps cross-referenced with $b=0$ T2-weighted images and a tractography atlas for accurate placement. ${ }^{18-20}$ A single region of interest was placed in the entire corpus callosum in the midsagittal plane for the interhemispheric fiber pathway. A 2-region-of-interest approach was used for studying projection and LAF pathways. Superior longitudinal fascicle, inferior fronto-occipital fascicle, and uncinate fascicle together constitute LAFs. ${ }^{16,17}$ The tracts intersecting the single region of interest or both ROIs in the case of LAF were constructed allowing tract-based measures. 


\begin{tabular}{|c|c|c|c|c|c|c|c|c|}
\hline & \multicolumn{4}{|c|}{ RRMS $(n=20 / 47)$} & \multicolumn{4}{|c|}{$\operatorname{CIS}(n=27 / 47)$} \\
\hline & RRMS & Control & MCIS & Control & PCISWOE & Control & PCISWE & Control \\
\hline No. of subjects & 20 & 20 & 9 & 9 & 9 & 9 & 9 & 9 \\
\hline Mean age at disease onset (yr) & 14.23 & NA & 12.99 & NA & 15.06 & NA & 9.37 & NA \\
\hline Range & $6.6-16.5$ & & 4.1-17.4 & & 13.9-18 & & $2.6-15.3$ & \\
\hline Mean age at the time of MRI (yr) & 15.92 & 15.925 & 13.58 & 13.58 & 15.58 & 15.52 & 9.74 & 9.4 \\
\hline Range & $8.4-18$ & $7.7-18$ & $4.1-18$ & $4-17.5$ & $14.5-18$ & $13-17$ & $2.6-15.8$ & $2-15$ \\
\hline Female sex (\%) & $75 \%$ & & $77.78 \%$ & & $66.67 \%$ & & $55.56 \%$ & \\
\hline \multicolumn{9}{|l|}{ Race } \\
\hline White & $75 \%$ & & $77.78 \%$ & & $66.67 \%$ & & $44.44 \%$ & \\
\hline Hispanic & $05 \%$ & & $00 \%$ & & $22.22 \%$ & & $11.11 \%$ & \\
\hline American black & $15 \%$ & & $11.11 \%$ & & $11.11 \%$ & & $22.22 \%$ & \\
\hline Asian & $05 \%$ & & $11.11 \%$ & & $00 \%$ & & $11.11 \%$ & \\
\hline $\begin{array}{l}\text { Mean disease duration from } 1 \text { st } S x \text { to } \\
\text { MRI analyzed }\end{array}$ & $2.11 \mathrm{yr}$ & NA & $0.27 \mathrm{yr}$ & NA & $0.37 \mathrm{yr}$ & NA & $0.1125 \mathrm{yr}$ & NA \\
\hline Mean EDSS & 1.625 & NA & 1.83 & NA & 1.38 & NA & 2.6 & NA \\
\hline No. of patients on DMT at the time of MRI & $15 / 20$ & NA & $00 / 10$ & NA & 02/09 & NA & $00 / 10$ & NA \\
\hline Avonex ${ }^{a}$ & $05 / 15$ & NA & $00 / 10$ & NA & $00 / 09$ & NA & $00 / 10$ & NA \\
\hline Rebifa & $04 / 15$ & NA & $00 / 10$ & NA & $01 / 09$ & NA & $00 / 10$ & NA \\
\hline Copaxone & $04 / 15$ & NA & $00 / 10$ & NA & $01 / 09$ & NA & $00 / 10$ & NA \\
\hline Multiple drugs & $02 / 15$ & NA & $00 / 10$ & NA & $00 / 09$ & NA & $00 / 10$ & NA \\
\hline No. of patients on monthly steroids & $05 / 20$ & NA & $00 / 10$ & NA & $00 / 09$ & NA & $00 / 10$ & NA \\
\hline $\begin{array}{l}\text { No. of patients on steroids within } 1 \text { month } \\
\text { of MRI }\end{array}$ & $05 / 20$ & NA & $06 / 10$ & NA & $02 / 09$ & NA & $10 / 10$ & NA \\
\hline
\end{tabular}

Note:-NA indicates not applicable; EDSS, Expanded Disability Status Scale; Sx = symptom; DMT, disease-modifying treatment.

a Interferon $\beta$-la.

${ }^{\mathrm{b}}$ Glatiramer acetate.

For NAWM, the identical ROIs were placed on color FA maps cross-referenced to $b=0 \mathrm{~T} 2$-weighted images to ensure that ROIs were placed in the NAWM. ${ }^{9}$ These ROIs were placed in the genu, anterior midbody, posterior midbody, and splenium of the corpus callosum; PLIC, cerebral peduncle; in the anatomic region to segment the superior longitudinal fascicle; and in the region at the level of anterior floor of the external capsule to segment the inferior fronto-occipital fascicle and uncinate fascicle. Tracts intersecting these ROIs were constructed. Any portion of the resulting tracts passing through regions of abnormal T2 signal on $b=0$ T2-weighted images were excluded. ADC and FA images were loaded into Track-Vis, and the mean ADC and FA in the specific ROIs and in the resulting tracts were recorded (On-line Fig 2).

The conventional MR imaging findings in each patient group were obtained by visual inspection under the direct supervision of an experienced pediatric neuroradiologist. The number of T2 lesions in the brain and spinal cord was manually counted by using T2-weighted MR images. For lesion location, T1-weighted and T2-weighted images were used, and these were verified with T2weighted FLAIR images. For defining lesion characteristics, T1weighted, T1 postgadolinium, and T2-weighted sequences were used.

\section{Statistical Analysis}

For each group (RRMS, MCIS, PCISWOE, and PCISWE), patients and matched controls were compared by using a paired $t$ test. DTI measures in patients with CIS were also considered as potential predictors of conversion to clinically definite RRMS by using a logistic regression model. In this analysis, the DTI measures were corrected by subtracting the matched control level to eliminate the residual effects from differences in scanners and protocol. The time from the first symptom to the last visit was controlled for as a potential confounder in the logistic regression model. Finally, the association between conversion and both the KIDMUS and Barkhof criteria was assessed by using the Fisher exact test. All statistical analysis was completed in the statistical package R (http://www.R-project.org).

\section{RESULTS}

\section{Demographic and Clinical Characteristics}

Baseline characteristics of all patients and matched controls are provided in Table 1, and an overview of patient cohorts is depicted in On-line Fig 1. Patients with PCISWE were, on average, younger, more likely to be male, and less likely to be white than other groups. Patients with MCIS had a mean follow-up of $1.72 \pm$ 0.91 years (range, $1.5-4.34$ years), and patients with PCISWE had a mean follow-up of period of $2.37 \pm 1.62$ years (range, $1-6.96$ years). Patients with PCISWOE were followed for a mean period of $2.07 \pm 1.33$ years (range, $2-6.33$ years), and 6/9 patients eventually developed clinically definite MS during this follow-up period. The remaining 21 patients with CIS did not experience a second demyelinating event or an MS-confirming event, neuromyelitis optica, or other chronic demyelinating diseases during their respective follow-up periods.

\section{Conventional MR Imaging Characteristics}

Conventional MR imaging T2 lesion numbers in the brain and spinal cord were assessed and are presented in Table 2. Patients with RRMS had a high T2 lesion load followed by those with PCISWOE, PCISWE, and MCIS. Most patients with MCIS had optic nerve involvement alone $(n=6)$. Patients with PCISWE mainly had subcortical lesions and lesions in the basal ganglia and thalamus. T2 lesions in patients with PCISWOE were mainly in the periventricular, subcortical, and infratentorial regions. Most 
Table 2: Conventional MRI features in patients with pediatric CIS and pediatric MS

\begin{tabular}{|c|c|c|c|c|}
\hline \multirow{2}{*}{$\begin{array}{l}\text { Conventional MRI } \\
\text { Features }\end{array}$} & \multicolumn{3}{|c|}{$\begin{array}{l}\text { MRI at the Time of } \\
\text { Presentation }\end{array}$} & \multirow{2}{*}{$\begin{array}{c}\text { MRI at the } \\
\text { Time of } \\
\text { Diagnosis } \\
\text { RRMS }\end{array}$} \\
\hline & MCIS & PCISWOE & PCISWE & \\
\hline \multicolumn{5}{|l|}{ Lesion location } \\
\hline Gray matter cortex & 0 & 0 & 2 & 3 \\
\hline BG and thalamus & 0 & 2 & 6 & 12 \\
\hline Subcortical & 0 & 5 & 8 & 19 \\
\hline Periventricular & 1 & 7 & 4 & 20 \\
\hline Perpendicular to CC & 0 & 2 & 2 & 16 \\
\hline Internal capsule & 0 & 1 & 2 & 7 \\
\hline Abutting the LV & 0 & 0 & 0 & 10 \\
\hline Cerebellum & 0 & 0 & 4 & 12 \\
\hline Brain stem & 0 & 4 & 4 & 12 \\
\hline Spinal cord & 2 & 3 & 0 & 13 \\
\hline Optic nerve & 6 & 0 & 2 & 0 \\
\hline $\begin{array}{c}\text { No. of } \mathrm{T} 2 \text { lesions in } \\
\text { the brain (range) }\end{array}$ & $0-1$ & $5-46$ & $1-22$ & $2-136$ \\
\hline $\begin{array}{c}\text { No. of T2 lesions in } \\
\text { the SC (range) }\end{array}$ & $0-2$ & $0-7$ & $0-7$ & $0-11$ \\
\hline \multicolumn{5}{|l|}{ Lesion characteristics } \\
\hline Well-defined lesions & & 4 & 1 & 16 \\
\hline Diffuse & 1 & 8 & 10 & 22 \\
\hline Ovoid & 0 & 7 & 4 & 20 \\
\hline Irregular-shaped & 0 & 6 & 9 & 19 \\
\hline Confluent & 0 & 1 & 1 & 6 \\
\hline Tumorlike & 1 & 0 & 4 & 5 \\
\hline Gd enhancement & 6 & 1 & 1 & 6 \\
\hline T1 black hole & 0 & 0 & 0 & 11 \\
\hline
\end{tabular}

Note:-BG indicates basal ganglia; LV, lateral ventricle; SC, spinal cord; Gd, gadolinium; CC, corpus callosum.

Table 3: DTI measures of white matter pathways in pediatric MS

\begin{tabular}{llll}
\hline & \multicolumn{1}{c}{ Patient } & Control & $P$ Value \\
\hline CC & & & \\
Tract mean ADC (SD) & $0.94(0.13)$ & $0.85(0.089)$ & $.0025^{\mathrm{b}}$ \\
Tract mean FA (SD) & $0.52(0.037)$ & $0.57(0.028)$ & $.0001^{\mathrm{b}}$ \\
PLIC & & & \\
Tract mean ADC (SD) & $0.73(0.058)$ & $0.70(0.059)$ & $.030^{\mathrm{b}}$ \\
$\quad$ Tract mean FA (SD) & $0.54(0.035)$ & $0.56(0.032)$ & $.020^{\mathrm{b}}$ \\
CP & & & \\
Tract mean ADC (SD) & $0.75(0.058)$ & $0.72(0.060)$ & $.0025^{\mathrm{b}}$ \\
Tract mean FA (SD) & $0.56(0.037)$ & $0.59(0.042)$ & $.037^{\mathrm{b}}$ \\
LAF & & & \\
Tract mean ADC (SD) & $0.81(0.083)$ & $0.76(0.064)$ & $.0054^{\mathrm{b}}$ \\
Tract mean FA (SD) & $0.44(0.034)$ & $0.47(0.031)$ & $.017^{\mathrm{b}}$ \\
\hline
\end{tabular}

Note:- $\mathrm{CP}$ indicates cerebral peduncle; $\mathrm{CC}$, corpus callosum.

${ }^{a} \mathrm{ADC}, \times 10^{-3}$.

b Statistically significant.

were either diffuse or ovoid in nature. Patients with RRMS had T2 lesions mainly in the subcortical region, perpendicular to the corpus callosum, and in the periventricular region. The presence of hypointense lesions on the T1 images was confined to the RRMS group.

\section{White Matter Pathway and NAWM Analysis in Patients with Pediatric MS}

In children with MS, tract-based mean ADC values were significantly increased in all tract systems, including callosal, projection pathways, and LAFs, compared with matched healthy controls (Table 3). Tract-based mean FA values were significantly decreased in pediatric patients with MS compared with matched
Table 4: DTI measures of NAWM in pediatric MS

\begin{tabular}{|c|c|c|c|}
\hline & Patients & Controls & $P$ Value \\
\hline \multicolumn{4}{|l|}{ Genu of CC } \\
\hline Mean ADC $( \pm S D)^{a}$ & $0.87(0.10)$ & $0.82(0.11)$ & .052 \\
\hline Mean FA ( \pm SD) & $0.53(0.050)$ & $0.56(0.051)$ & .098 \\
\hline \multicolumn{4}{|l|}{ Splenium of CC } \\
\hline Mean ADC $( \pm S D)^{a}$ & $0.91(0.13)$ & $0.79(0.095)$ & $.0010^{b}$ \\
\hline Mean FA ( \pm SD) & $0.58(0.073)$ & $0.65(0.052)$ & $.0003^{b}$ \\
\hline \multicolumn{4}{|l|}{$\mathrm{AMB}$ of $\mathrm{CC}$} \\
\hline Mean ADC $( \pm S D)^{a}$ & $0.89(0.12)$ & $0.79(0.085)$ & $.0005^{b}$ \\
\hline Mean FA ( \pm SD) & $0.52(0.057)$ & $0.57(0.051)$ & $.0053^{b}$ \\
\hline \multicolumn{4}{|l|}{$\mathrm{PMB}$ of $\mathrm{CC}$} \\
\hline Mean ADC ( \pm SD) & $0.93(0.12)$ & $0.84(0.11)$ & $.011^{b}$ \\
\hline Mean FA ( \pm SD) & $0.53(0.051)$ & $0.57(0.051)$ & $.032^{b}$ \\
\hline \multicolumn{4}{|l|}{ PLIC } \\
\hline Mean ADC $( \pm S D)^{a}$ & $0.72(0.053)$ & $0.71(0.059)$ & .38 \\
\hline Mean FA ( \pm SD) & $0.56(0.039)$ & $0.56(0.031)$ & .77 \\
\hline \multicolumn{4}{|l|}{$\mathrm{CP}$} \\
\hline Mean ADC $( \pm S D)^{a}$ & $0.74(0.061)$ & $0.73(0.065)$ & .29 \\
\hline Mean FA ( \pm SD) & $0.58(0.041)$ & $0.59(0.044)$ & .18 \\
\hline \multicolumn{4}{|l|}{ SLF } \\
\hline Mean ADC $( \pm S D)^{a}$ & $0.76(0.072)$ & $0.71(0.070)$ & $.0020^{b}$ \\
\hline Mean FA ( \pm SD) & $0.47(0.042)$ & $0.52(0.043)$ & $.00004^{b}$ \\
\hline \multicolumn{4}{|l|}{ IFOF and UF } \\
\hline Mean ADC $( \pm S D)^{a}$ & $0.82(0.081)$ & $0.77(0.067)$ & $.0006^{b}$ \\
\hline Mean FA ( \pm SD) & $0.46(0.038)$ & $0.49(0.033)$ & $.0095^{b}$ \\
\hline \multicolumn{4}{|l|}{ All NAWM } \\
\hline Mean ADC $( \pm S D)^{a}$ & $0.81(0.062)$ & $0.76(0.066)$ & $.0008^{b}$ \\
\hline Mean FA ( \pm SD) & $0.53(0.033)$ & $0.57(0.028)$ & $.0002^{b}$ \\
\hline
\end{tabular}

Note:-AMB indicates anterior midbody; PMB, posterior midbody; SLF, superior longitudinal fascicle; UF, uncinate fascicle; IFOF, inferior fronto-occipital fascicle; CP, cerebral peduncle; $C$, corpus callosum.

a $\mathrm{ADC}, \times 10^{-3}$

b Statistically significant.

controls in all tract systems. Similar results were obtained by using region-of-interest analysis for mean ADC and mean FA.

Tract-based mean ADC was significantly increased in pediatric patients with MS in all tracts passing through ROIs of NAWM (Table 4), including the splenium of the corpus callosum, anterior midbody and posterior midbody of the corpus callosum, association fibers, superior longitudinal fascicle, inferior fronto-occipital fascicle and uncinate fascicle, and an aggregate of all tracts passing through NAWM. Similarly, tract-based mean FA values were significantly decreased in pediatric patients with MS in a similar distribution (Table 4). However, callosal fibers in the genu of the corpus callosum and projection fibers in the NAWM showed no significant difference, but increased mean ADC and decreased mean FA were observed compared with those in matched controls.

\section{White Matter Pathway and NAWM Analysis in Patients with CIS}

The mean timeframe between MR imaging and the first demyelinating event in the MCIS group was 0.27 years (range, 1-6 months). In the PCISWOE group, it varied from 0.25 to 2 years (range, 3-24 months) and the mean time was 0.37 years. In the PCISWE group, the timeframe was shorter: it varied from 0.1 to 0.5 years ( 14 days to 6 months), and the mean time was 0.113 years.

In general, patients in all CIS groups showed a nonsignificant trend toward an increased mean ADC and a decreased mean FA in all the white matter pathways compared with healthy controls 
Table 5: DTI measures of white matter pathways in pediatric CIS-baseline MRI

\begin{tabular}{|c|c|c|c|c|c|c|c|c|c|}
\hline & \multicolumn{3}{|c|}{ MCIS } & \multicolumn{3}{|c|}{ PCISWOE } & \multicolumn{3}{|c|}{ PCISWE } \\
\hline & Patients & Controls & $\begin{array}{c}P \\
\text { Value }\end{array}$ & Patients & Controls & $\begin{array}{c}P \\
\text { Value }\end{array}$ & Patients & Controls & $\begin{array}{c}P \\
\text { Value }\end{array}$ \\
\hline \multicolumn{10}{|l|}{$\mathrm{CC}$} \\
\hline Mean ADC $( \pm$ SD $)$ & $0.87(0.086)$ & $0.84(0.073)$ & .46 & $0.95(0.11)$ & $0.89(0.056)$ & .10 & $1.01(0.12)$ & $1.02(0.093)$ & .67 \\
\hline Mean FA ( \pm SD) & $0.53(0.042)$ & $0.56(0.033)$ & .20 & $0.55(0.030)$ & $0.58(0.049)$ & .15 & $0.56(0.059)$ & $0.55(0.039)$ & .87 \\
\hline \multicolumn{10}{|l|}{ CP } \\
\hline Mean ADC $( \pm S D)$ & $0.72(0.062)$ & $0.72(0.056)$ & .75 & $0.74(0.060)$ & $0.73(0.048)$ & .19 & $0.80(0.044)$ & $0.79(0.030)$ & .61 \\
\hline Mean FA ( \pm SD) & $0.55(0.032)$ & $0.59(0.040)$ & $.045^{\mathrm{a}}$ & $0.59(0.052)$ & $0.60(0.063)$ & .67 & $0.60(0.073)$ & $0.60(0.059)$ & .97 \\
\hline \multicolumn{10}{|l|}{ PLIC } \\
\hline Mean ADC $( \pm S D)$ & $0.72(0.058)$ & $0.71(0.051)$ & .68 & $0.73(0.063)$ & $0.72(0.053)$ & .33 & $0.76(0.030)$ & $0.76(0.036)$ & .77 \\
\hline Mean FA $( \pm S D)$ & $0.53(0.013)$ & $0.56(0.041)$ & .067 & $0.57(0.053)$ & $0.58(0.052)$ & .66 & $0.59(0.087)$ & $0.60(0.039)$ & .65 \\
\hline \multicolumn{10}{|l|}{ LAF } \\
\hline Mean ADC ( \pm SD) & $0.78(0.051)$ & $0.73(0.051)$ & $.018^{\mathrm{a}}$ & $0.79(0.057)$ & $0.77(0.051)$ & .33 & $0.84(0.038)$ & $0.84(0.035)$ & .73 \\
\hline Mean FA ( \pm SD) & $0.45(0.021)$ & $0.46(0.037)$ & .41 & $0.46(0.018)$ & $0.49(0.039)$ & $.041^{\mathrm{a}}$ & $0.47(0.083)$ & $0.46(0.051)$ & .65 \\
\hline
\end{tabular}

Note:-CP indicates cerebral peduncle; CC, corpus callosum.

a Statistically significant.

(Table 5). The only exceptions were a significant effect on ADC in the LAF for patients with MCIS, on FA in the cerebral peduncle for patients with MCIS, and in the LAFs for patients with PCISWOE. These results would not remain significant after accounting for multiple comparisons.

In general, there was no difference in mean $\mathrm{ADC}$ or $\mathrm{FA}$ in the entire white matter pathway confined to NAWM in all the MCIS, PCISWOE, and PCISWE groups at the time of the CIS event compared with controls (On-line Table). The only exceptions were significant decreases in FA (but no change in ADC) in the LAF in PCISWOE. Similarly, in patients with MCIS, there was a significant difference in the mean ADC but not in the FA in the LAF. As seen before, these results would not remain significant after accounting for multiple comparisons.

\section{Prediction of Conversion to MS}

To assess the potential predictive value of our DTI measures in patients with CIS, we compared those who converted to MS with those who did not convert during follow-up. None of the DTI measures in white matter pathways or in NAWM in patients with CIS were significantly associated with conversion to RRMS in univariate or multivariate models ( $P>.05$ in all models). Because some of the scans were performed acutely, we performed an additional analysis to assess data from MRI obtained $>14$ days after the acute event; by using these data, we also found no associations with conversion to MS. Most interesting, all the converters to MS had PCISWOE at onset; therefore, an additional analysis was completed to determine the predictive ability of DTI measures in this subgroup. However, no measures were significantly associated with conversion in this analysis $(P>.05$ in all models).

The Barkhof and KIDMUS criteria were also investigated as potential predictors because these had been assessed in recent publications. ${ }^{11,12}$ Using logistic regression, fulfillment of the KIDMUS criteria was significantly associated with conversion to MS $(P=.006)$, but the Barkhof criteria were not $(P=.14)$. The Barkhof criteria demonstrated $60 \%$ sensitivity and $76 \%$ specificity, while the KIDMUS criteria demonstrated 50\% sensitivity and $100 \%$ specificity (Table 6).

\begin{tabular}{|c|c|c|}
\hline & $\begin{array}{l}\text { Patients with } \\
\text { CIS Who } \\
\text { Converted } \\
\text { to MS }\end{array}$ & $\begin{array}{c}\text { Patients with } \\
\text { CIS Who } \\
\text { Did Not Convert } \\
\text { to MS }\end{array}$ \\
\hline \multicolumn{3}{|l|}{ Barkhof criteria } \\
\hline Satisfied Barkhof criteria ${ }^{a}$ & 4 & 5 \\
\hline Did not satisfy Barkhof criteria ${ }^{a}$ & 2 & 16 \\
\hline \multicolumn{3}{|l|}{ KIDMUS criteria } \\
\hline Satisfied KIDMUS criteria ${ }^{\mathrm{b}}$ & 3 & 0 \\
\hline Did not satisfy KIDMUS criteriab & 3 & 21 \\
\hline
\end{tabular}

${ }^{a}$ Sensitivity $=60 \%$; specificity $=76.1 \%$.

${ }^{\mathrm{b}}$ Sensitivity $=50 \%$; specificity $=100 \%$.

\section{DISCUSSION}

The main objective of the study was to identify different trends in quantitative DTI measures of mean ADC and mean FA of 3 major white matter pathways in pediatric MS and different subcategories of CIS in children. We found significantly increased mean ADC and decreased mean FA in all white matter pathways assessed containing lesioned and nonlesioned tracts and NAWM containing nonlesioned tracts of pediatric patients with MS. Similar to findings in adult MS studies ${ }^{21-23}$ and our previous study in pediatric MS, ${ }^{9}$ diffusion abnormalities were again noted in the callosal fibers, projection fibers, and association fiber pathways in pediatric patients with MS in our separate and unique cohort. We did not find significant involvement of these structures in the MCIS and PCISWOE groups. The only exceptions were significant associations in FA in the LAF in patients with PCISWOE and MCIS. There was no trend of increased mean ADC and decreased mean FA of these structures in the PCISWE group. Most important, baseline DTI measures of FA and ADC in major pathways in patients with CIS were not predictive of MS.

Limitations of this study were differences in DTI parameters and the time between scanning and the initial demyelinating event, mainly due to the retrospective nature of the study; however, we accounted for this limitation by matching the controls with specific scanner subtypes and DTI acquisition protocol. The MRI of the patients with MS was not performed within 30 days of the demyelinating event, whereas the MRI of the patients with CIS was within 30 days of the demyelinating event; however, repeating 
the imaging of these patients at a later time point did not make any difference in the results.

DTI and tractography studies in adult CIS have shown conflicting results regarding involvement of NAWM. Patients with optic neuritis demonstrated anisotropic changes in the splenium of the corpus callosum, correlating with future risk of MS. ${ }^{18}$ Others found occult damage in both normal-appearing gray matter and NAWM in CIS, but to a lesser extent than in RRMS. ${ }^{19}$ In patients with CIS, diffusion MR imaging did not detect alterations in the NAWM of patients, but diffusion MR imaging abnormalities in the NAWM became apparent after 1 year. ${ }^{20}$ Most interesting, studies contrasting adult-onset acute disseminated encephalomyelitis and MS found higher mean diffusivity in the NAWM of patients with MS, presumably reflecting widespread tissue destruction seen in MS, which was not present in adult acute disseminated encephalomyelitis. ${ }^{24}$ Our findings that children with acute demyelinating syndromes consistent with either monofocal CIS, polyfocal CIS with encephalopathy, or polyfocal CIS without encephalopathy did not demonstrate significant anisotropic abnormalities in the NAWM suggest that these diffuse pathologic processes are not present acutely at the time of clinical disease onset. Our definition of NAWM excluded overt focal lesions but included dirty-appearing white matter, ${ }^{25}$ and further studies are required to dissociate these entities in pediatric MS. Similar to our work, a recent study found significantly decreased mean FA in the NAWM callosal fibers and NAWM corticospinal tracts and significantly increased MD in the NAWM corticospinal tracts in adult MS compared with the pediatric MS/CIS and adult CIS groups. ${ }^{26}$ Pediatric MS and CIS may represent an earlier form of MS, closer to the true onset of disease, with consequently less pathologic burden than adult MS.

Children with acute demyelinating syndromes, including CIS and acute disseminated encephalomyelitis, may develop MS in the future. In most cases, children converted within a 2-year period of follow-up. ${ }^{8,10}$ Previously published studies have suggested that patients with nonencephalopathic presentations carried a higher risk of developing MS than those with encephalopathy. In our study, baseline DTI measures of FA and ADC in major pathways in patients with CIS were not predictive of MS. We found that the strongest predictor of conversion to MS was presentation with a PCISWOE. None of the children with other CIS presentations, including PCISWE and MCIS, converted in the follow-up period. In our study, the fulfillment of both the KIDMUS and Barkhof criteria at baseline was a specific but insensitive predictor of MS, suggesting that broader, less stringent predictive MR imaging criteria need to be developed. ${ }^{27}$

\section{CONCLUSIONS}

Children with MS demonstrated significant anisotropic changes in all 3 white matter pathways examined. There was no trend toward changes in ADC and FA values in patients with PCISWE, suggesting that diffuse processes do not develop with time in this disease. The statistically nonsignificant trend toward increasing ADC and decreasing FA suggests that the period between PCISWOE and established MS represents a "window of opportunity" in which diffuse processes and potentially progressive disability may be prevented. Several studies have demonstrated that the administration of disease-modifying treatments at the time of CIS in adults delays disease progression. ${ }^{28,29}$ Because of the present difficulty in identifying children at highest risk for MS after a first event, disease-modifying treatments are currently not administered to children in this situation. Predictive biomarkers to identify children with a CIS event who are at highest risk for MS may allow the early initiation of disease-modifying treatment and prevention of progressive disability.

Disclosures: Tanuja Chitnis—RELATED: consulting fees received from Biogen-Idec, EMD-Serono, Teva Neurosciences, Novartis, and Bayer. The other authors report no conflicts of interest.

\section{REFERENCES}

1. Chitnis T, Glanz B, Jaffin S, et al. Demographics of pediatric-onset multiple sclerosis in an MS center population from the Northeastern United States. Mult Scler 2009;15:627-31

2. Boiko A, Vorobeychik G, Paty D, et al. Early onset multiple sclerosis: a longitudinal study. Neurology 2002;59:1006-10

3. Ghezzi A, Deplano V, Faroni J, et al. Multiple sclerosis in childhood: clinical features of 149 cases. Mult Scler 1997;3:43-46

4. Renoux C, Vukusic S, Mikaeloff Y, et al. Natural history of multiple sclerosis with childhood onset. N Engl J Med 2007;356:2603-13

5. Gorman MP, Healy BC, Polgar-Turcsanyi M, et al. Increased relapse rate in pediatric-onset compared with adult-onset multiple sclerosis. Arch Neurol 2009;66:54-59

6. MacAllister WS, Belman AL, Milazzo M, et al. Cognitive functioning in children and adolescents with multiple sclerosis. Neurology 2005;64:1422-25

7. Amato MP, Goretti B, Ghezzi A, et al. Cognitive and psychosocial features of childhood and juvenile MS. Neurology 2008;70:1891-97

8. Hahn CD, Shroff MM, Blaser SI, et al. MRI criteria for multiple sclerosis: evaluation in a pediatric cohort. Neurology 2004;62: 806-08

9. Vishwas M, Chitnis T, Pienaar R, et al. Tract-based analysis of callosal, projection and association pathways in pediatric patients with multiple sclerosis: a preliminary study. AJNR Am J Neuroradiol 2010;31:121-28

10. Mikaeloff Y, Suissa S, Vallee L, et al. First episode of acute CNS inflammatory demyelination in childhood: prognostic factors for multiple sclerosis and disability. J Pediatr 2004;144:246-52

11. Mikaeloff $Y$, Adamsbaum C, Husson B, et al. MRI prognostic factors for relapse after acute CNS inflammatory demyelination in childhood. Brain 2004;127:1942-47

12. Neuteboom RF, Boon M, Catsman Berrevoets CE, et al. Prognostic factors after a first attack of inflammatory CNS demyelination in children. Neurology 2008;71:967-73

13. Krupp LB, Banwell B, Tenembaum S. Consensus definitions proposed for pediatric multiple sclerosis and related disorders. Neurology 2007;68:S7-12

14. Mori S, van Zijl PC. Fiber tracking: principles and strategies-a technical review. NMR Biomed 2002;15:468-80

15. Wakana S, Jiang H, Nagae-Poetscher LM, et al. Fiber tract-based atlas of human white matter anatomy. Radiology 2004;230:77-87

16. Catani M, Howard RJ, Pajevic $S$, et al. Virtual in vivo interactive dissection of white matter fasciculi in the human brain. Neuroimage 2002;17:77-94

17. Catani M, Thiebaut de Schotten M. A diffusion tensor imaging tractography atlas for virtual in vivo dissections. Cortex 2008;44: 1105-32

18. Bester M, Heesen C, Schippling S, et al. Early anisotropy changes in the corpus callosum of patients with optic neuritis. Neuroradiology 2008;50:549-57

19. Yu CS, Lin FC, Liu Y, et al. Histogram analysis of diffusion measures in clinically isolated syndromes and relapsing-remitting multiple sclerosis. Eur J Radiol 2008;68:328-34 
20. Caramia F, Pantano P, Di Legge S, et al. A longitudinal study of MR diffusion changes in normal appearing white matter of patients with early multiple sclerosis. Magn Reson Imaging 2002;20:383-88

21. Werring DJ, Clark CA, Barker GJ, et al. Diffusion tensor imaging of lesions and normal-appearing white matter in multiple sclerosis. Neurology 1999;52:1626-32

22. Cercignani $M$, Inglese $M$, Pagani E, et al. Mean diffusivity and fractional anisotropy histograms of patients with multiple sclerosis. AJNR Am J Neuroradiol 2001;22:952-58

23. Hasan KM, Gupta RK, Santos RM, et al. Diffusion tensor fractional anisotropy of the normal-appearing seven segments of the corpus callosum in healthy adults and relapsing-remitting multiple sclerosis patients. J Magn Reson Imaging 2005;21:735-43

24. Inglese M, Salvi F, Iannucci G, et al. Magnetization transfer and diffusion tensor MR imaging of acute disseminated encephalomyelitis. AJNR Am J Neuroradiol 2002;23:267-72
25. Vrenken H, Seewann A, Knol DL, et al. Diffusely abnormal white matter in progressive multiple sclerosis: in vivo quantitative MR imaging characterization and comparison between disease types. AJNR Am J Neuroradiol 2010;31:541-48

26. Rocca MA, Absinta M, Moiola L, et al. Functional and structural connectivity of the motor network in pediatric and adult-onset relapsing-remitting multiple sclerosis. Radiology 2010;254:541-50

27. Chitnis T, Pirko I. Sensitivity vs specificity: progress and pitfalls in defining MRI criteria for pediatric MS. Neurology 2009;72:952-53

28. Kappos L, Freedman MS, Polman CH, et al. Effect of early versus delayed interferon beta- $1 \mathrm{~b}$ treatment on disability after a first clinical event suggestive of multiple sclerosis: a 3-year follow-up analysis of the BENEFIT study. Lancet 2007;370:389-97

29. Kinkel RP, Kollman C, O'Connor P, et al. IM interferon beta-1a delays definite multiple sclerosis 5 years after a first demyelinating event. Neurology 2006;66:678-84 